\title{
ADAPTAÇÃO DO MÉTODO TEAM-BASED LEARNING PARA O MODELO MEDIADO POR TECNOLOGIAS VIRTUAIS DE APRENDIZAGEM
}

\author{
Adaptation of the Team-Based Learning method to the model guided by virtual \\ learning technologies
}

\author{
Juleimar Soares Coelho de Amorim (iD https://orcid.org/0000-0003-3218-1769 \\ Instituto Federal do Rio de Janeiro \\ E-mail: juleimar.amorim@ifri.edu.br
}

Ricardo Esteves Kneipp (iD https://orcid.org/0000-0002-6611-6722

Instituto Federal do Rio de Janeiro

E-mail: ricardo.kneipp@ifri.edu.br

\section{Resumo}

O método Team-Based Learning (TBL) foi escolhido para este trabalho como estratégia de ensino virtual por sua versatilidade e proposição do aprendizado ser colaborativa em equipe, embora essa experiência ainda não tenha sido discutida na literatura. $O$ objetivo foi apresentar e descrever uma proposta do método de adaptação do ensino TBL em formato virtual. Foi apresentada uma adequação ao homônimo TBL já utilizado como metodologia ativa em ensino presencial, o qual visa descrever e analisar, de forma integrada, fundamentos para adaptação e inclusão nas práticas docentes, possibilitando aproveitamentos ou empregos como recursos ou ferramentas para ampliar, inovar e agregar nos processos de ensino-aprendizagem dos estudantes e docentes. Trata-se de um estudo teórico-reflexivo que propõe uma adaptação do TBL virtual, por meio do pacote Google for Education. O público escolhido para essa proposta é de alunos do curso de graduação e pós-graduação, docentes e gestores. Foi realizada uma análise descritiva sobre a viabilidade das atividades educacionais do $T B L$ virtual, conforme os recursos do ambiente de aprendizagem. Em seguida, foi realizada uma análise de viabilidade por meio da matriz SWOT, a partir do levantamento teórico de evidências sobre o método $T B L$ e o ensino a distância e virtual. Os resultados permitiram discutir que a novidade pode contribuir para solidificar as metodologias ativas no ensino a distância e foram identificadas revisão do planejamento e papel docente, a adequação curricular e institucional e a evasão foram discutidos como problemas que ainda precisam ser superados.

Palavras-chave: Educação a distância. Disseminação de tecnologia educacional. Tecnologia educacional.

\begin{abstract}
The Team-Based Learning (TBL) method was chosen for this work as a virtual teaching strategy because of its versatility and the proposition of learning to be collaborative in a team, although this experience has not yet been discussed in the literature. The objective was to present and describe a proposal for the method of adapting TBL in virtual format. An adaptation to the namesake TBL already used as an active methodology in face-to-face teaching was presented, which aims to describe and analyze, in an integrated way, the fundamentals, and inclusion in teaching practices, enabling uses or usage as resources or tools to expand, innovate and aggregate in the teachinglearning processes of students and teachers. This is a theoretical-reflective study that proposes an
\end{abstract}


adaptation of virtual TBL, through the Google for Education package. The public chosen for this proposal is undergraduate and post graduate students, teachers, and managers. A descriptive analysis was carried out on the viability of the academic activities of the TBL, according to the resources of the learning environment. Then, a feasibility analysis was performed using the SWOT matrix, based on the theoretical survey of evidence about the TBL method and e-learning. The results made it possible to discuss that this new way may contribute to solidify the active methodologies in distance education and a review of the planning and teaching role, curricular and institutional adequacy and dropout are discussed as problems that still need to be overcome.

Keywords: Distance learning. Dissemination of educational technology. Educational Technology.

\section{Introdução}

As modificações que ocorreram nos últimos anos na sociedade provenientes da disponibilidade das fontes de informação, da globalização, do uso da tecnologia e das necessidades, demandas e exigências do mercado de trabalho, além das políticas públicas de ensino vigentes no país, repercutiram numa reflexão sobre a dinâmica do processo de aprendizagem e criaram a necessidade de um novo olhar para a formação profissional no ensino superior (CURREY et al., 2015). Os métodos apoiados na aprendizagem por descoberta significativa têm buscado modificar 0 ensino por parte do professor "invertendo" as aulas em relação às tradicionais no que diz respeito: (i) ao foco do processo de ensino, que passa a ser centrado no aluno ao invés do professor, (ii) ao primeiro contato do discente com o conteúdo a ser estudado e (iii) à capacidade crítica de discutir sobre o conteúdo aprendido (HRYNCHAK, 2012).

O método Team-Based Learning (TBL), ou Aprendizado Baseado em Equipe, prioriza o tempo em sala de aula para dirimir dúvidas e resolver problemas com os colegas. Em contraposição ao tradicional, em que é em sala de aula que o aluno se depara com o conhecimento a ser estudado e depois, sozinho em casa, se dedica à resolução de hiatos que venham a surgir (HRYNCHAK, 2012; CURREY et al., 2015). Há evidências que o TBL é uma metodologia de aprendizagem ativa (o discente enquanto sujeito da construção do seu conhecimento, autonomia, autogestão do processo de formação) (MITRE et al., 2008; OUELLETTE, BLOUNT, 2015) que melhora o desempenho do aluno (ANDERSEN et al., 2011; ANWAR et al., 2012; KOLES et al., 2010; LETASSY et al., 2008), desenvolve o trabalho em equipe (HUNT et al., 2003), o pensamento crítico (McINERNEY et al., 2003) e tem relação com a satisfação com a aprendizagem (CLARK et al., 2008; MASTERS, 2012; WILLETT et al., 2011).

A estratégia Instrução de Pares favorece uma comunicação interpares intercalada com a resolução de conceitos próprios do conteúdo de aprendizagem tem ganhado consistência científica e é adotada em metodologias ativas (FAGEN, 2002). Apesar das metodologias ativas terem expandido no cenário brasileiro de ensino, ainda é incipiente a divulgação de experiências exitosas que possam estimular e capacitar gestores e docentes na aplicação prática (ANDERSEN et al., 2011; HRYNCHACK et al., 2012; LARA, ROSATTI, JOVETTA, 2016). Experiências anteriores para os cursos da Saúde foram descritas no ensino da Ética em curso de Saúde Pública (VAN DER PUTTEN, 2017), de Ginecologia e Obstetrícia na Enfermagem (MOORES-DAVIS et al., 2015) e de Fisioterapia (AMORIM et al, 2019; MICHAELSEN et al., 2004).

À medida que habilidades como comunicação e trabalho em equipe estão se tornando cada vez mais importantes para uma carreira na graduação, metodologias que nutrem essas habilidades, como TBL, estão se tornando mais valiosas em cursos de ensino superior. Assim, é imperioso preparar os alunos para a prática colaborativa desde sua formação, mas também fortalecer a incorporação das Tecnologias de Informação e 
Comunicação (TIC) para a atuação profissional (GOUDOURIS et al., 2013). Alcançar esse objetivo, perpassa a transformação do trabalho docente, que precisa incorporar esses preceitos, tornar-se integrado a outras disciplinas e conhecimentos, como no cenário da Era digital (ABREU et al., 2020). A evolução da internet facilita cada vez mais o acesso à formação, com uma produção de conteúdo de diversas fontes (CUNHA, 2005; RAYMOND et al., 2016).

Os estudos anteriores têm mostrado que as aulas na modalidade a distância podem fornecer mais flexibilidade do que aulas presenciais (SCHOONHEIM et al., 2014; CENDAN, 2012; BOLING et al., 2012; HILL et al., 2009). Muitos programas de graduação e pós-graduação oferecem cursos selecionados ou componentes de cursos online para fornecer uma oportunidade de ensino a distância (COLLINS et al., 2017; PHILIPS et al., 2016; KING, 2015). Com programas de aprendizagem flexíveis, como Ensino a Distância (EaD) (RAYMOND et al., 2016), e pedagogias de aprendizagem ativa, como TBL (HRYNCHAK, 2012), se tornando cada vez mais populares na educação em saúde, é possível que surja uma necessidade maior para o TBL a distância. Propomos que o ensino virtual pode ser uma estratégia que permita atividades envolventes de TBL para ensino a distância.

Os cursos na modalidade a distância consistem em momentos de interação por meio de tecnologias informacionais e prevê interação entre os professores, alunos e equipe administrativa. A Plataforma Moodle, entre outras, geralmente é adotada como mediadora do processo ensino-aprendizagem e repositório de material de aula (ABREU, 2020; BOLING, 2012). Contudo, apesar dos avanços nos formatos das aulas e da apresentação dos conteúdos e avaliações, a metodologia de ensino ainda continua orientada à direção professor-aluno, em que a partir da disponibilização das aulas e seus materiais pelo professor os alunos têm o primeiro contato com o conteúdo de aprendizagem (ABREU, 2020; BOLING, 2012; O'DOHERTY, 2018).

Considerando que a metodologia ativa por meio da problematização e do aprendizado baseado em equipe inverte a ordem de apresentação do conteúdo de aprendizagem, aluno-professor, compreender as nuances de sua incorporação nos cursos EaD é desafiador para a prática docente. Apesar de estudos sobre o método de ensino baseado em equipe e que incorpore as tecnologias informacionais terem sido publicados previamente, especialmente no Brasil, este artigo discute a importância de estabelecer adequações pelo professor que ministra as aulas à distância e em conhecer adequações e potencialidades do método em relação ao presencial (O'DOHERTY, 2018; ONCU, 2011; SCHOONHEIM, 2014).

A modalidade $\mathrm{EaD}$ constitui um grupo de modalidade que requer repensar a educação, uma vez que adaptações na sua incorporação se relaciona na prática de trabalho do professor, nas habilidades e competências do discente e infraestrutura por parte da Instituição. Em adição à intencionalidade pedagógica, também busca-se continuar oportunizando acesso à aprendizagem a toda comunidade, independente da presencialidade, buscando consonância com a Educação $4.0^{1}$ (termo adotado para expressar a cultura digital e a inovação tecnológica na educação) por intermédio das tecnologias digitais. Assim, a aprendizagem pode ser extrapolada para além da sala de aula presencial, gerando ainda mais significado para o aprender. Após uma revisão da literatura, como hipótese, tem-se que conhecer as adequações do método TBL virtual poderá favorecer a sua incorporação na prática docente. O objetivo do estudo

\footnotetext{
${ }^{1}$ termo adotado para expressar a cultura digital e a inovação tecnológica na educação
} 
foi apresentar e descrever uma proposta do método de ensino Team-Based Learning em formato virtual de ensino.

\section{Desenvolvimento}

\section{i) Delineamento e público-alvo}

Este manuscrito é uma proposta de adaptação e segue um delineamento descritivo. Apesar do método TBL ser direcionado a alunos do ensino médio até a pósgraduação, há evidências de melhores resultados no ensino de graduação ou pósgraduação (COLLINS et al., 2017; PHILIPS et al., 2016; KING, 2015). De igual modo, o método apresenta uma versatilidade quanto aos conteúdos a serem abordados, podendo ser facilmente adaptado pelos docentes para disciplinas teóricas, práticas ou teórico-práticas. Entretanto, disciplinas com carga horária elevada e predominantemente conteudistas são preferenciais para a utilização do método (HRYNCHAK, 2012). Assim, o público escolhido para essa proposta é de docentes e discentes de graduação em saúde.

\section{ii) Descrição do produto - Atividade educacional TBL virtual}

O produto que é apresentado se refere à atividade educacional TBL em formato virtual (TBL virtual). Originalmente, o método TBL é ofertado em formato de sessões, às quais são organizadas em três grandes etapas e suas subetapas, com delimitação de tempo (MICHAELSEN et al., 2008; SIBLEY, 2014).

I) A primeira etapa refere-se a etapa pré-classe, denominada de Preparação, em que cada estudante é orientado a iniciar a sessão com contato prévio ao conteúdo por meio da leitura de textos, assistir vídeos, exercícios de simulação, entrevista, conferência, experimentos e buscas livres. Nessa etapa, do ponto de vista pedagógico, é importante garantir ao estudante a lista de objetivos de aprendizagem e referências mínimas para estudo. Isso exige do docente a gestão e o planejamento de modo a possibilitar o primeiro contato do aluno com o conteúdo por alternativas próprias dele, mas por apoio ou orientação do docente, que passa a ser um mediador.

II) Em seguida, em classe, a segunda fase refere-se a Garantia do Preparo por meio do Teste de Preparo Individual (TPi), Teste de Preparo em Equipe (TPe), Apelação e Exposição Dialogada com feedback do professor. Os alunos iniciam a sessão em sala de aula com uma avaliação rápida individual sobre o conteúdo orientado previamente, na sequência essa mesma avaliação é discutida entre os membros da equipe e possíveis apelações sobre o tema são direcionadas ao professor (mediador, facilitador ou tutor). O instrutor expõe a temática com a proposta de dirimir as dúvidas, provocar reflexões e internalização do conhecimento. Nessa etapa, o planejamento docente requer preparo dos testes e preparação da aula de forma tão flexível que responda aos anseios e necessidades dos alunos em função dos objetivos de aprendizagem estabelecidos. Por parte dos discentes, requer assiduidade, pontualidade e responsabilidade consigo e com os demais membros da equipe.

III) Por fim, ainda em classe, inicia-se a terceira fase de Aplicação dos Conceitos por meio de um problema significativo, com mesmo tema e relatos simultâneos a partir das estratégias avaliativas como questões estruturadas ou 
resolução de casos. Os integrantes devem chegar a um consenso sobre a melhor solução a partir das opções fornecidas, e simultaneamente relatar sua decisão para toda a classe. $O$ instrutor, então, facilita uma discussão entre as equipes nas respostas que escolheram e seus fundamentos. Essa última etapa poderá extrapolar os muros da sala de aula, com possibilidade de ser finalizada em outro momento, uma vez que há a proposição de apresentar conclusões ou soluções para o tema estudado. É importante que ao final da sessão, os estudantes tenham dirimidos todas as dúvidas, mas não necessariamente esgotado o assunto. O papel docente, nesse caso, é de incorporar suas experiências do mundo do trabalho técnico para propor problemas e desafios interessantes aos alunos.

O tempo estipulado para cada fase é díspar e dependerá da organização da disciplina na grade curricular do curso, o que pode durar alguns minutos ou dias. $\mathrm{Na}$ fase de Preparação, os alunos podem ser orientados a conduzir seus estudos com intervalo de uma semana, por exemplo, e na aula seguinte já iniciar com a Fase 2 (Garantia do Preparo). Para o momento intraclasse, estima-se entre 50-90 minutos de sessão, tanto para a Fase 2 quanto a fase de Aplicação. Cerca de 60 minutos pode ser suficiente para conclusão da terceira etapa.

Trata-se de um recurso digital, que será mediado por uma tecnologia da informação e comunicação à serviço da gestão educacional, destinado à promover o ensino por meio da colaboração em equipe. Seu desenvolvimento poderá ser aberto à toda comunidade acadêmica e garante maior apoio em ferramentas de metodologias ativas ao docente, tanto que atua na $\mathrm{EaD}$ quanto em cursos presenciais com carga horária de ensino virtual e/ou em Atividades Pedagógicas Não Presenciais.

Entendendo que por ser um espaço de aprendizagem formal, para essa elaboração, os princípios da Política Nacional de Ciência, Tecnologia e Inovação (MINISTÉRIO DA SAÚDE, 2008) serão adotados buscando (i) inclusão - inserção de usuários, docentes e discentes da produção técnico-científica; e, (ii) complementaridade entre as lógicas da comunicação, tecnologia e ensino. Além disso, esses requisitos da tecnologia foram incorporados à prática moderna da educação, tais como (i) colaboração; (ii) metodologia inovadora; (iii) popularização do ensino; e, (iv) espaços de aprendizagem. Portanto, esse produto, visa em primeira instância, oferecer experiências de ensino-aprendizagem que extrapolem os meios físicos e/ou curriculares da formação em saúde. Percebe-se utilidade particular desse produto, já que diante do contexto atual de ensino, a aprendizagem poderá ser extrapolada para além da sala de aula física e os discentes poderão se relacionar por meio de aprendizado colaborativo.

\section{iii) Definição do ambiente virtual de aprendizagem}

Atualmente, são inúmeros os ambientes de aprendizagem que estão disponíveis no cenário da EaD, alguns são privativos a instituições, outros são públicos, pagos e gratuitos. Os aplicativos da ferramenta Google for Education tais como, gmail, Classroom, Drive, Docs, planilha, apresentações e google Forms podem servir de apoio ao professor no processo pedagógico e é, atualmente, a mais utilizada em instituições públicas de ensino (AMORIM et al., 2019; ANWAR et al., 2012; HRYNCHACK, 2012; ANDERSEN et al., 2011; CLARK et al., 2008). Trata-se de uma solução tecnológica que envolve seus usuários e foi desenvolvida para facilitar a educação em diferentes níveis, a qualquer hora e a partir de qualquer dispositivo conectado à internet. A característica marcante é que todo conteúdo inserido na 
plataforma pode ser acessado a qualquer momento, do local onde o estudante ou o professor estiver, a partir de qualquer dispositivo, uma vez que $100 \%$ do material didático - livros, trabalhos, documentos, e-mails, agendas e tarefas - fica salvo automaticamente em nuvem (Cloud Computing). Ainda, possibilita uma maior interação entre os próprios alunos no desenvolvimento e acompanhamento de atividades acadêmicas, como trabalhos em equipe, como no caso do TBL. Isso é possível porque esses aplicativos citados acima foram criados exatamente para serem usados de forma mais participativa e colaborativa, pois possibilitam alterações e edições em tempo real, propiciando a realização de tarefas e afazeres de modo compartilhado.

A seguir, serão apresentados os recursos utilizados dentro da Google for Education considerados como infraestrutura mínima para as sessões do TBL. Essa ferramenta foi escolhida por conveniência e por ser disponibilizada pelo Instituto Federal de Educação, Ciência e Tecnologia do Rio de Janeiro (IFRJ) em todos seus cursos de graduação e pós-graduação.

(1) Classroom é um pacote de ferramentas para produtividade em educação e está disponível gratuitamente a qualquer pessoa que utilize o Gmail, Google Drive e Documentos Google. Por meio desse ambiente, o professor poderá organizar as sessões previamente e deixá-las disponíveis a qualquer tempo para os alunos. Essa poderá ser a sala principal de debates, discussões, definição das tarefas e resolução dos problemas elencados no TBL.

(2) Forms é uma ferramenta de formulários onde o professor pode construir suas pesquisas, enquetes, coletar opiniões, e-mails e informações de contato de forma simples e rápida. Dentro do google forms, existem os tipos de questões que são utilizadas e estão disponíveis, tais como, resposta curta, parágrafo, múltipla escolha, caixas de seleção, lista suspensa, upload de arquivo, escala linear, grade de múltipla escolha, grade da caixa de seleção, data e horário. Para os tipos de questões existe a possibilidade de oferecer a resposta para que seja feita a correção e de tornar as perguntas obrigatórias, de modo que o questionário só poderá ser enviado se todas as questões obrigatórias estiverem respondidas. O formulário criado poderá ser disponibilizado através de um endereço eletrônico onde será respondido pelos alunos e as respostas aparecerão imediatamente na página do Google Forms do professor que 0 criou. Poderá ser utilizado como fonte de avaliação, pesquisa e acompanhamento dos estudantes, pois no contexto de ambiente virtual de aprendizagem, o professor consegue fazer um questionário individualizado, que exibe quantas questões cada estudante acertou. Por seu caráter de formulação de questões, esse recurso será útil para formação das questões de aprendizagem (testes), seja individual ou em equipe. A estratégia de questionários online pode ser interessante pois criam atitude correta de aprendizado, podem ser eficientes guias para a explanação, como no caso do $T B L$ e eles aumentam a curiosidade sobre as respostas. Não é obrigatório sempre ser somativo, pois o objetivo não é avaliar, mas fornecer um meio para que os estudantes recapitulem o que já aprenderam e testem seus conhecimentos. Quando eles percebem que não sabem algumas respostas, são motivados a ficar atentos para descobrir a resposta quando o assunto for abordado em equipe ou com o professor. O importante é que esses questionários devem ser desafiadores, e não necessariamente complexos.

(3) Docs é um serviço que permite o acesso, a criação, e edição de documentos no smartphone, tablet ou computador em qualquer lugar. Permite que se trabalhe junto e 
simultaneamente o mesmo documento. Ela funciona diretamente da web, totalmente gratuita até o limite de $15 \mathrm{~Gb}$ de dados, para utilizá-la basta fazer login com a senha da conta do Google no site https://docs.google.com. Está organizado em três partes: Documentos, Planilhas e Apresentações. Enquanto o primeiro é um editor de texto, o segundo serve para criar e editar planilhas e, por fim, o terceiro tem como função a criação de apresentações de slides. Para a proposta do TBL, esse recurso será importante em casos de sínteses mais elaboradas, ou conclusões das sessões com caráter descritivas.

(4) Drive é um serviço de disco virtual, oferecendo 15 GB de espaço gratuito para seus usuários. Permite o armazenamento de arquivos na nuvem do Google e possui aplicativos para sincronização. Os arquivos armazenados na Google Drive, podem ser compartilhados com os professores e alunos através da conta da Google. É possível editar usuários com quem compartilhar os arquivos, além de definir o nível de permissão de cada pessoa (visualização, edição ou comentários). Tanto instrutor quanto os estudantes e equipes poderão utilizar essa ferramenta para reunir documentos da própria turma, textos para leitura, vídeos, entre outros, como uma espécie de repositório.

(5) Meet será a interface para videoconferência e videochamadas, as quais possibilitam ensino e aprendizagem de forma síncrona. O professor pode abrir uma sala virtual e iniciar sua exposição a partir da chamada de áudio e vídeo. É possível projetar a tela para compartilhar uma apresentação e os estudantes podem interagir com seus microfones abertos ou pelo chat. Por meio desse dispositivo, a atividade síncrona pode ser do tipo aula expositiva-dialogada. A etapa de exposição do conteúdo de aprendizagem e explanação, bem como o momento de interação entre estudantes e professor, poderá ser facilitado por meio dessa ferramenta de forma a reforçar conceitos, debater as teorias ou apresentar produtos gerados com a temática da sessão.

Além do pacote da Google, algumas outras ferramentas virtuais são importantes para garantir adequação aos fundamentos do TBL, o que está relacionado à:

(a) Divisão das equipes por randomização: O randomizationplain.com é uma plataforma que permite gerar grupos (equipes) aleatoriamente. No caso do TBL, será utilizado para a formação das equipes com até cinco participantes. Os membros podem se auto randomizar em equipes, inscrevendo-se em pequenos times para participar.

(b) Feedback pós sessão: Ao final de cada sessão, os estudantes são incentivados a fornecer uma avaliação breve da própria sessão, do conteúdo, da equipe, do professor ou até mesmo da disciplina. A Mentimeter (https://www.mentimeter.com/) é uma plataforma fácil para obter informações em tempo real de equipes remotas e alunos on-line com enquetes ao vivo, questionários, nuvens de palavras, perguntas e respostas. Por meio de uma única palavra, os participantes podem fornecer informações significativas e tomarem consciência sobre o processo de aprendizagem do qual fizeram ou fazem parte. A resposta do participante pode ocorrer com foco na avaliação sobre o que aprendeu com relação a um conceito, sobre a nota numérica resultante de um processo, sobre o seu comportamento, sobre um trabalho em equipe ou até mesmo sobre uma autoavaliação que o aluno realizou.

\section{iv) Recursos educacionais abertos - repositórios}


Recursos Educacionais Abertos tem como objetivo na aula o acesso à publicação online de coleções nacionais e temáticas de livros acadêmicos em formato digital, tanto para estudantes quanto docentes (Venturini, 2014). Em cursos da saúde, algumas ofertas de livros e textos didáticos são oferecidos pelo Scientific Electronic Library Online - SciELO Livros (http://books.scielo.org/), Pubmed Book (https://www.ncbi.nlm.nih.gov/books/) $\quad$ e $\quad$ Portal Capes (http://www.periodicos.capes.gov.br/) e são legíveis nos leitores de ebooks, tablets, smartphones e telas de computador. De igual modo, existem bases de dados para busca de livre acesso de bibliografias, citações e resumos de artigos de investigação em literatura da área da saúde. As bases de dados da Medical Literature Analysis and Retrieval System Online - Medline (https://pubmed.ncbi.nlm.nih.gov/), Physiotherapy Evidence Database - PEDro (https://pedro.org.au/) e Literatura Latino-americana e do Caribe em Ciências da Saúde - Lilacs (https://lilacs.bvsalud.org/) fornecem artigos de investigação na área da saúde. Esses recursos educacionais são fontes primordiais para as sessões do $T B L$ e requerem uma apresentação, manuseio e treinamento antes mesmo de todas as sessões. Por meio desses recursos, os alunos farão suas buscas e serão as ferramentas de primeiro contato do estudante com o conteúdo.

A partir da proposta de adequação do TBL presencial para o virtual, a Figura 1 apresenta as etapas e subetapas de uma sessão, as ferramentas tecnológicas e caracterização da formatação em relação à constituição do trabalho, ao tempo e o processo de evolução.

Figura 1 - Modelo síntese virtual para Team-Based Learning

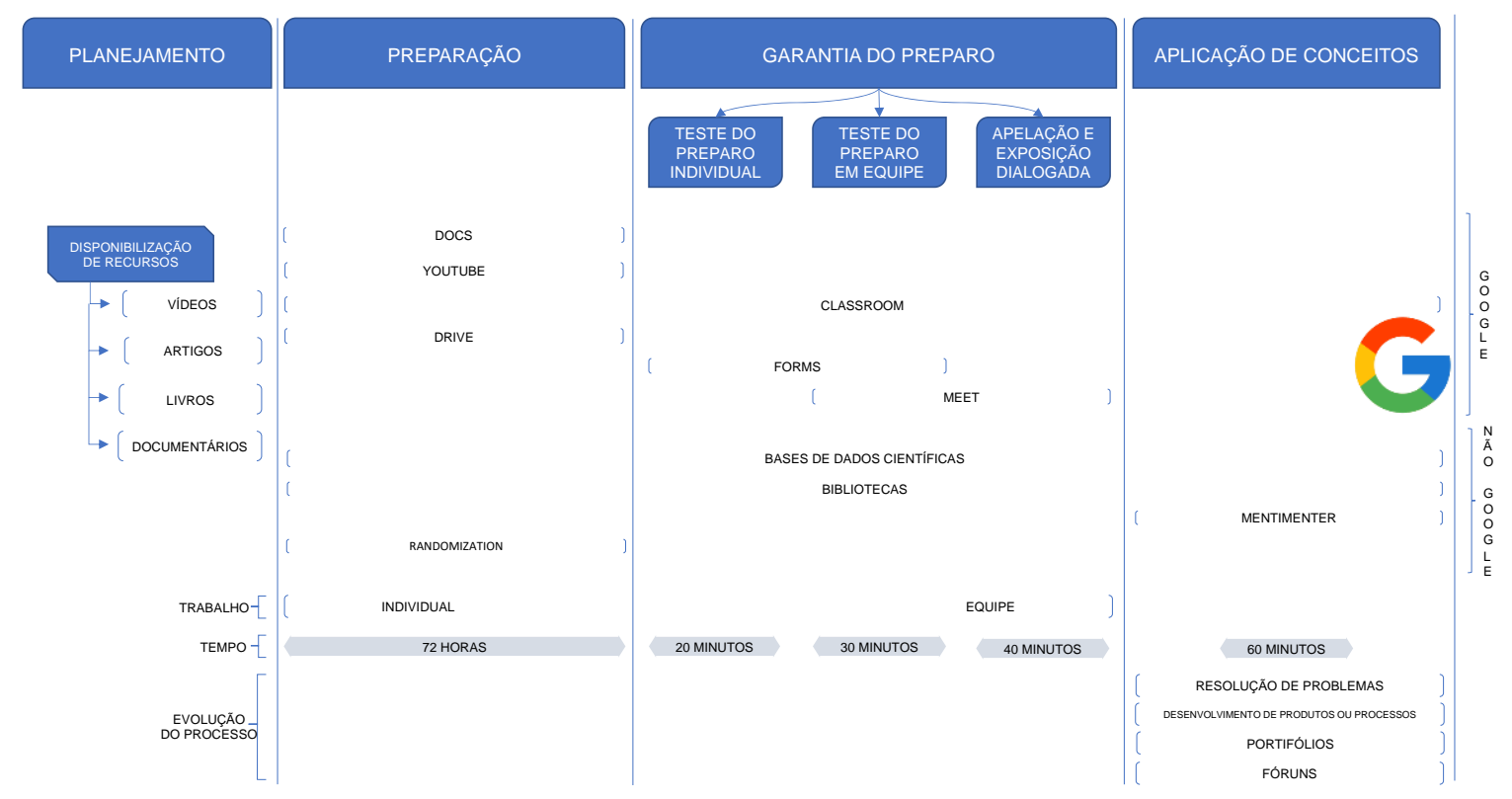

Fonte: Elaboração própria

\section{v) Análise de viabilidade}

Foi realizada uma busca na literatura sobre a incorporação da metodologia $T B L$ em cursos de graduação e pós-graduação, seja em ensino à distância ou presencial, e sobre as condições relacionadas à infraestrutura institucional na oferta de cursos ou disciplinas no formato de TBL virtual. Para o seu desenvolvimento, são elencados 
elementos metodológicos considerados como subtemas que delimitam o campo de conhecimento; ou seja, aquelas evidências que favorecem a discussão da viabilidade.

A partir dessa busca, foram selecionados elementos para compor a análise de viabilidade por meio da Matriz SWOT (em português, FOFA), organizados em ambiente interno (Strengths - Forças; Weaknesses - Fraquezas) e externo (Opportunities - Oportunidades e Threats - Ameaças). As análises derivadas por meio da matriz permitiram, mesmo que teoricamente, compreender as possibilidades $e$ limitações da tomada de decisão sobre a adaptação do método para o virtual. A análise foi organizada em núcleos temáticos de compreensão que permitem julgar a respeito adaptação do método $T B L$ virtual na prática docente, a saber: interação social, presença social, relação professor-aluno, tradução para a prática docente $e$ incorporação institucional do TBL. Por fim, permeando toda matriz SOWT, foram identificados elementos estratégicos para a decisão da adaptação. Os elementos selecionados são apresentados no Quadro 1. 
Quadro 1 - Análise de viabilidade para adaptação do TBL virtual, de acordo com elementos da Matriz SWOT.

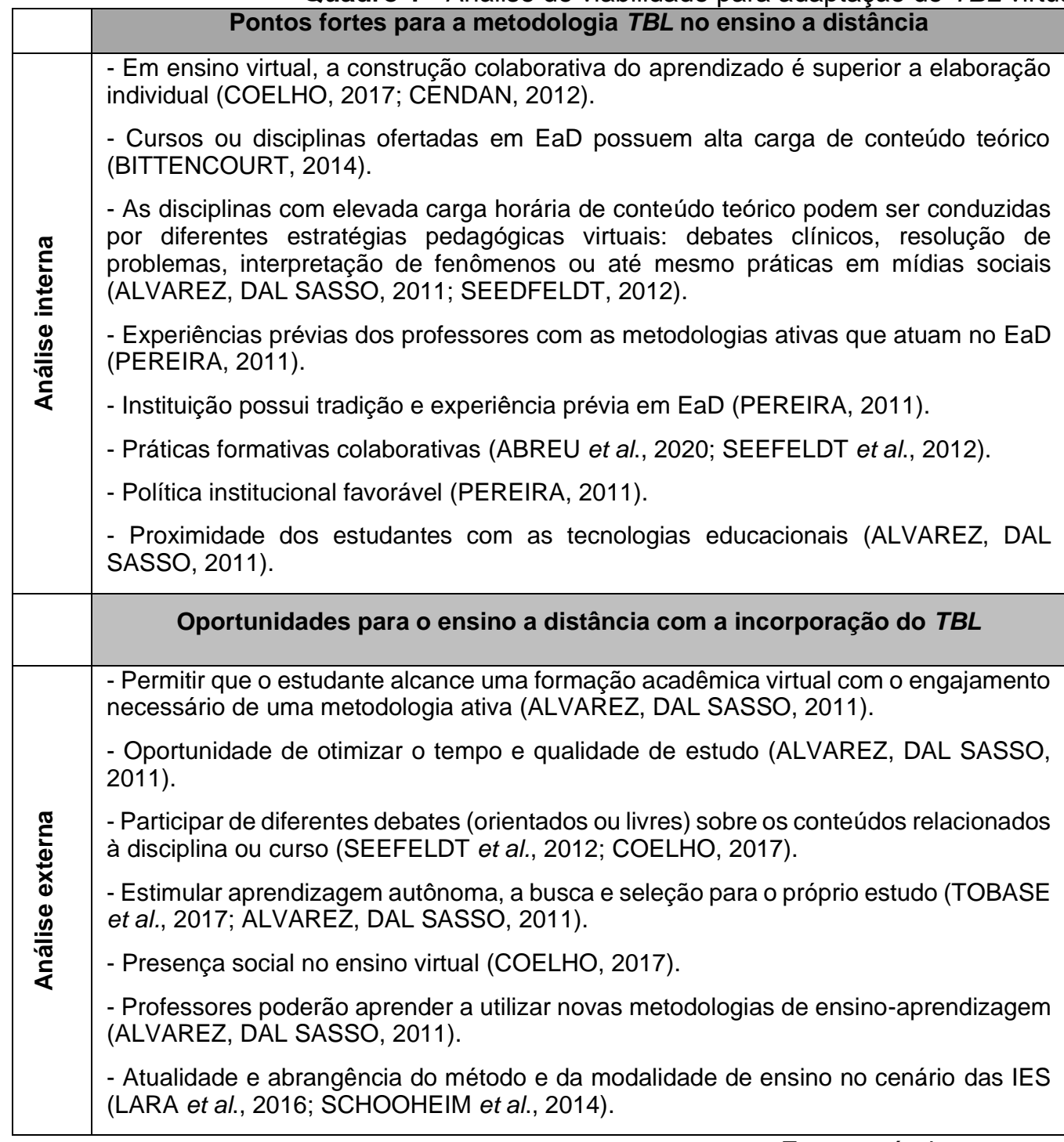

- Disciplinas ou cursos exclusivamente práticos não são recomendadas para o método TBL e nem para o virtual (LAU et al., 2017; GOUDOURIS et al., 2013; O'DOHERTY et al., 2018; CURREY et al., 2015; HRYNCHAK, 2012; REIMSCHISEL et al., 2017).

- Disciplinas ou cursos cujo conteúdo teórico é predominantemente baseado em memorização, reconhecimento, identificação não são recomendadas para a metodologia TBL e nem para o virtual (LAU et al., 2017; GOUDOURIS et al., 2013; O'DOHERTY et al., 2018; CURREY et al., 2015; HRYNCHAK, 2012; REIMSCHISEL et al., 2017).

- Impossibilidade de avaliação e acompanhamento do conteúdo em disciplinas ou cursos que exigem visita técnica (EL TANTAWI et al., 2013).

- Conceitos pré-concebidos sobre EaD, e-Learning e virtual ou resistência com o TBL (AMORIM et al., 2019; OLIVEIRA, 2017).

- Inexistência de um ambiente virtual de aprendizagem institucional próprio (ALVAREZ, DAL SASSO, 2011).

\section{Dificuldades ou ameaças para o ensino a distância com a} incorporação do $T B L$

- Desenvolvimento de ferramentas próprias da instituição para a construção de disciplinas ou cursos (disciplinas) virtuais no formato necessário para o $T B L$ e o desenvolvimento de ambientes de aprendizagem virtual pode ser caro (SCHOONHEIM et al., 2014).

- Interação e presença social entre estudantes do curso (COELHO, 2017).

- Formação em qualificação de docentes com a metodologia ativa em ensino virtual (ABREU et al., 2020).

- Evasão em EaD, APNP ou e-Learning (AMORIM et al., 2019).

- Requer cuidado desde a concepção até a implementação e acompanhamento dos participantes (TOBASE et al., 2017; SCHOONHEIM et al., 2014).

\footnotetext{
Fonte: próprios autores (2020)
} 
Este manuscrito apresentou como proposta a entrega de uma adaptação tecnológica para facilitar a implementação do método $T B L$ virtual, a partir da utilização das ferramentas gratuitas da Google for Education. Dado o uso frequente da Google, essa oferta aplica o modelo em cursos ou disciplinas em educação superior e distintos contextos educativos da EaD. O ensino por meio de plataformas virtuais tem cada vez mais expandido nas instituições de educação, é relativamente econômico e pode fornecer experiências para muitos alunos ao mesmo tempo (MAUÉS et al., 2015). Como exemplos, na área da saúde tem sido cada vez mais comum imersão em realidade virtual, sistemas educacionais em ambientes virtuais, ensino por plataformas online e até mesmo aplicativos de ensino-aprendizagem para os diferentes cursos de graduação em Farmácia, Medicina, Fisioterapia, Enfermagem e Odontologia (CENDAN, 2012; EL TANTAWI et al., 2013; SCHOONHEIM et al., 2014; SASSO, 2006; LAU et al., 2017). Apesar desse avanço nas ferramentas tecnológicas, os modelos de ensino ainda requerem adequações, sobretudo no que tange a interação (homem-máquina, estudante-estudante e estudante-professor), resolução de problemas virtuais reais e do próprio currículo. Também ainda são escassas aquelas tecnologias que permitem a sociabilidade necessária para a metodologia $T B L$ (CENDAN, 2012).

A utilização da análise de viabilidade por meio da Matriz SWOT produziu resultados que ampliaram a compreensão para a gestão e processos da formação acadêmica para o TBL virtual. A melhora no entendimento sobre os pontos positivos e negativos do processo possibilitou uma visão do cenário de modo a elencar as ameaças e oportunidades em relação à adaptação proposta. Dessa forma, essa matriz contribuiu na compreensão dos pontos favoráveis e desfavoráveis de uma implementação e possibilitou ainda refletir sobre a necessidade de uma verificação empírica de sua incorporação nos cursos EaD, mas também a lacuna sobre o TBL para o processo de atuação de docentes nesta modalidade de ensino. Evidência prévia que utilizou essa análise de cenário, reporta que sua simplicidade permite subsidiar a gestão e o planejamento para posicionar e verificar as condições de incorporação da metodologia de ensino (PEREIRA, 2011).

A matriz permitiu entender que determinada característica do método TBL virtual considerada como força ou fraqueza é relativa, subjetiva e potencialmente alterável, designadamente na medida em que se podem verificar ao longo de sua implementação. Em outras palavras, as análises apresentadas devem ser consideradas com cautela, pois se trata de uma técnica de julgamento passível de subjetividades e os fatores elencados podem variar entre os elementos constitutivos da matriz conforme a interpretação individual. Contudo, em outra estratégia avaliativa, estudo sobre a adequação do design instrucional no desenvolvimento de curso online para alunos de enfermagem, Tobase et al (2017) avaliaram o curso sobre Suporte Básico de Vida por meio do modelo ADDIE (Analysis, Design, Development, Implementation e Evaluation). Os autores concluíram que o design instrucional se mostrou adequado ao desenvolvimento do curso, tanto por especialistas quanto estudantes, principalmente em relação a qualidade e quantidade do conteúdo, interatividade, liberdade de navegação e aprendizado. Assim como a matriz SOWT, a ADDIE permite visão geral do processo educativo e ambos favorecem decisões para ajustes. Infere-se, portanto, que a adequação das estratégias na construção do conhecimento por meio do TBL virtual, bem como a sua aplicação na prática, amplia 0 alcance de recursos educacionais.

Interação social 
A interação social tem sido apontada como elemento-chave para a aprendizagem e para a construção colaborativa do conhecimento em ambientes virtuais (COELHO, 2017; BOLING et al., 2012; HILL et al., 2009). No entanto, pode ser desafiador garantir que os alunos experimentem o mesmo nível de envolvimento em aulas virtuais que fariam em presenciais. A teoria da presença social descreve até que ponto os participantes sentem que estão interagindo com pessoas reais quando estão online. No entanto, o e-Learning foi descrito como "um meio frio" pelos alunos (KEAR, 2010; COELHO, 2017), sugerindo um baixo nível de presença social. A presença social online é árdua, mesmo com o uso de fóruns e plataformas síncronas como salas de chat e videoconferência. Contudo, programas de ensino têm implementado cursos TBL online (MORRINSON et al., 2011; PALSOLÉ, 2008) sugerindo que há necessidade e oportunidade de estratégias que possibilitem o mesmo nível de engajamento para o TBL a distância que existe com o $T B L$ presencial.

\section{Presença Social}

A matriz SWOT indica oportunidades e desafios na implementação da EaD e do $T B L$ virtual que concernem sobre o conceito de presença social. Segundo Coelho e colaboradores (2017), os métodos de ensino em ambiente virtual requerem transpor as práticas de entrega de material autoinstrutivo e videoconferências (transmissão mecanicista de conteúdo) para atividades síncronas de interação (produção colaborativa de conhecimento). Ancorado na teoria sociocultural de Vygotsky, Oncu e Cakir (2011) defendem a colaboração virtual como propulsora do aumento no volume e qualidade do envolvimento de estudantes, bem como o engajamento e habilidade de pensar criticamente. Apesar dessas urgências nos currículos EaD, Woo e Reeves (2007) julgam que é um revés manter a interação no ambiente virtual quando comparado aos contextos presenciais, considerando a separação, no tempo e no espaço, provocada pelo uso (síncrono e assíncrono) da tecnologia. Para eles, a interação deve ser significativa de tal forma a estimular a curiosidade intelectual dos estudantes, envolvê-los em atividades produtivas de ensino e influenciar diretamente sua aprendizagem, o que inclui ações de diálogo, negociação (interna e socialmente), argumentação de pontos de vista, agregação de novas ideias e oferta de novas perspectivas a discussões de problemas reais (WOO, REEVES, 2007).

\section{Relação professor-aluno}

Algumas limitações e ameaças merecem ser exploradas para minimizar hiatos na relação professor-aluno e na relação com a EaD por meio do $T B L$. Recentemente, Amorim et al. (2019) analisou o método TBL presencial e identificou que o tempo de dedicação para uma sessão e o cansaço experimentado pelos estudantes são variáveis negativas. A metodologia por si só consome muito tempo para realização das atividades, o que é base para outra queixa de que a frequência semanal de tarefas leva à exaustão. Para os estudantes, o método exige pontualidade, assiduidade e esforço para superar dificuldades na interação e comunicação, o que parece ser mais exaustivo cognitivamente. A metodologia não é propositiva nas atividades rotineiras teóricas ao modo pergunta e resposta, leitura de casos clínicos ou produção de trabalhos acadêmicos. Justamente nesse âmbito, o TBL busca produzir, pedagogicamente, certo desconforto com o que se sabe, um incômodo ante a necessidade de saber para agir. Este processo de aprendizagem pretende mobilizar

(1)


e desafiar os estudantes para a busca e a abertura para o novo e para a ação (REIMSCHISEL et al., 2017).

\section{Tradução para a prática docente}

Outra crítica se refere à metodologia de aula. $O$ ensino por meio do $T B L$ exige esforço em acompanhar e estimular a discussão a partir de objetivos de aprendizagem. A metodologia ativa requer conhecimento e habilidades do docente que vão além de diversidade de técnicas de ensino, mas também a carga de trabalho deve ser ajustada pois estão implicados nesse processo o preparo exames em quantitativo maior, a mediação nas discussões entre as equipes e na preparação da metodologia em si, além da exposição dialogada de praxe. Guedes et al. (2013) observaram que professores de ensino e práticas da Fisioterapia utilizaram uma gama variada de recursos instrucionais, como multimídia, bonecos, dinâmicas, apresentação de trabalhos, aulas práticas, estágio curricular e discussão de caso clínico. Porém, predominantemente, observou-se um enfoque na metodologia tradicional, eminentemente transmissiva e, novamente, se faz necessário um processo de mudança da educação capaz de romper com estruturas estabelecidas e modelos de ensino enraizados. Somente após essa transposição, realmente os currículos proporcionarão a capacidade de formar profissionais de saúde autônomos com senso de coletividade, que admitam uma prática pedagógica ética, crítica, reflexiva e, por isso, transformadora, ultrapassando os limites do treinamento puramente técnico, inscrito na dialética da ação-reflexão-ação (PARK et al., 2015). Enquanto docente, é imperioso o estabelecimento de um diálogo customizado com os estudantes, conhecer as diferentes práticas e as dificuldades no aprendizado discorrido nos momentos do trabalho em equipe, auxiliando-os no processo de construção do conhecimento a partir da reflexão deles (conjunto de estudos, experiências e vivências) e identificando fendas para ativação de movimentos de vicissitudes. Ao mesmo tempo, o duelo também deve se permear pelo desenvolvimento da grupalidade, da coesão entre os componentes do grupo, a partir de elementos para uma cartografia conjunta. Desenvolve-se assim o eixo da coletividade, que favorece a construção dos saberes a partir das trocas de experiência e das reflexões com apoio do material teórico em um curso.

\section{Incorporação institucional do TBL}

Por fim, para a instituição, a incorporação do $T B L$ requer planejamento e gestão para um currículo centrado na problematização, o que demanda esforço coletivo para o aprendizado interdisciplinar, muito além da diversidade de técnicas de ensino em sala de aula. A depender da concepção pedagógica subjacente ao curso $T B L$ virtual, pode ser conflituoso propor estudos independentes e atividades colaborativas, entretanto, o pressuposto fundamental do TBL é o equilíbrio entre essas propostas, e o ambiente virtual de aprendizagem não as tornam mutuamente excludentes (ONRUBIA et al., 2010). O TBL virtual permite, nesse caso, integrar a colaboração e a tecnologia e vai de encontro a Aprendizagem Colaborativa Apoiada por Computador - CSCL (Computer-Supported Collaborative Learning) (ONRUBIA et al., 2010).

Enquanto outros estudos objetivam desenvolver ferramentas tecnológicas para diversas experiências em EAD (SASSO, 2006; SCHIBECl et al., 2008; SEEFELDT et al., 2012; EL TANTAWI et al., 2013; SCHOONHEIM et al., 2014), a presente proposta

1


contribui para a discussão sobre o desenvolvimento de metodologias de ensino capazes de romper os limites e as barreiras da presença social, interação e colaboração no ambiente virtual. A proposta de adaptar o método $T B L$ para sessões virtuais pretende estimular a autonomia do estudante, favorece o processo de aprendizagem autogerida, de maneira autoinstrucional, aliando os pressupostos da Andragogia e da Teoria da Aprendizagem Significativa às tecnologias educacionais, em razão do perfil dos estudantes que buscam os cursos virtuais ou a distância (LEIGH et al., 2015).

Esse debate teórico poderá estimular novos delineamentos de diferentes abordagens para se compreender as experiências apreendidas no processo ensino-aprendizagem e o impacto do método na formação de estudantes. Sugere-se como futuras pesquisas uma análise de viabilidade com especialistas e estudantes, estudos experimentais do $T B L$ virtual, a avaliação da satisfação e desempenho no plano de trabalho dos docentes, investigação das habilidades e competências relacionadas à comunicação escrita e oral, atitude e planejamento profissional.

\section{Considerações Finais}

Este trabalho apresentou uma proposta de modelo tecnológico para o aprendizado baseado em equipe, aqui denominado TBL virtual. O objetivo foi descrever como o suporte mediado por tecnologias da informação e comunicação, por meio da Google for Education, permitiu adaptar o TBL homônimo existente, a ser considerado em futuras implementações em disciplinas de diferentes níveis de formação no ensino superior. Esta proposta de inversão da sala de aula, do presencial para o virtual e do primeiro contato com o professor para o primeiro contato pelo aluno, pode ser uma novidade e contribui para solidificar as metodologias ativas no EaD.

O trabalho apresentou, a partir de uma revisão da literatura, uma análise de viabilidade e a estratégia tecnológica para incorporação por Instituições de Ensino, especialmente na saúde. Apesar de propositivo, limitações relacionadas ao planejamento e revisão do papel docente, adequação curricular e institucional e a evasão são discutidos como problemas que ainda precisam ser superados, principalmente na sobre a formação do professor para metodologia ativa no EAD. Na discussão, foram incorporados os antecedentes de experiências de implantação da TBL e do EAD no ensino superior, seguidos da apresentação do modelo TBL virtual como uma recomendação tecnológica para esta metodologia. $O$ debate sobre as relações de ensinoaprendizado oportunizado expressam uma conquista, uma vez que tais discussões são escassas nos cursos por ensino a distância, mas também um desafio, dada a carência de investimentos na formação do professor.

As transformações sociais, tecnológicas e educacionais estão impondo mudanças substanciais no modo de viabilizar acesso ao Ensino Superior. Novas soluções têm sido requeridas e não será oportuno interromper as atividades de ensino durante anos no país. Quanto maior a clareza acerca dos aspectos que devem orientar as decisões a serem tomadas nas Instituições de Ensino Superior, maiores as chances de seguirmos caminhos orientados, pela lucidez do conhecimento já produzido e pela adequada caracterização das condições existentes nas instituições, para estudantes e professores.

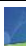




\section{Referências}

ABREU, E. T.; NOVAES, M. A.; ZARRO, M. I. M. Desafios na formação de professores para atuação na EAD. Rev. Paidéi@. Unimes Virtual, Santos, v.12, n.21, 2020. Disponível: https://periodicos.unimesvirtual.com.br/index.php/paideia/index Acesso em: 19 out. 2020.

ALVAREZ, A. G.; DAL SASSO, G. T. M. Aplicação de objeto virtual de aprendizagem, para avaliação simulada de dor aguda, em estudantes de enfermagem. Revista Latino-Americana Enfermagem, Ribeirão Preto, v.19, n.2, p. 9, 2011. Disponível em: https://www.scielo.br/j/rlae/a/4bcpsBnpxzC5SXKcwQjb3bf/?format=pdf\&lang=pt Acesso em: 20 jul. 2021

AMORIM, J. S. C. et al. Team-based learning in Physical Therapy undergraduate course: experiment report. Fisioterapia em Movimento, Curitiba, v.32, p. e003246, 2019. Disponível em: http://www.scielo.br/scielo.php?script=sci_arttext\&pid=S010351502019000100239\&lng=en\&nrm=iso. Acesso em: 11 out. 2020.

ANDERSEN, E. A et al. Implementing team-based learning in large classes: nurse educators' experiences. International Journal Nursing Education Scholarsh, Berkeley,. v.8, n.1., 2011. Disponível em: /j/ijnes.2011.8.issue-1/1548923X.2197/1548-923X.2197.xml. Acesso em: 10 out 2020.

ANWAR, K. et al. Comparing the efficacy of team based learning strategies in a problem based learning curriculum. APMIS: acta pathologica, microbiologica, et imunnologica Scandinavica, Copenhagen, v.20, n.9, p. 718-723, 2012. Disponível em: https://onlinelibrary.wiley.com/doi/10.1111/j.1600-0463.2012.02897.x Acesso em: 20 jul. 2021

BITTENCOURT, I. M.; MERCADO, L. P. L. Evasão nos cursos na modalidade de educação a distância: estudo de caso do Curso Piloto de Administração da UFAL/UAB. Ensaio: avaliação e Políticas Públicas em Educação, Rio de Janeiro, v.

22, n. 83, p. 465-504, 2014. Disponível em: https://revistas.cesgranrio.org.br/index.php/ensaio/article/view/296 Acesso em 20 jul. 2020

BOLING, E. C. et al. Cutting the distance in distance education: perspectives on what promote positive, online learning experience. Internet and Higher Education, v. 15, n. 2, p. 118-126, 2012 . Disponível em: https://www.sciencedirect.com/science/article/pii/S109675161100090X Acesso em: 20 jul. 2021

CENDAN, J.; LOK, B. The use of virtual patients in medical school curricula. Advances in Physiology Education, Rockville, v. 36, n. 1, p. 48-53, 2012. Disponível em: https://www.ncbi.nlm.nih.gov/pmc/articles/PMC3776425/ Acesso em: 20 jul. 2021

CLARK, M. C. et al. Team-based learning in an undergraduate nursing course. Journal Nursing Education, New York, v.47, n.3, p.111-117, 2008. Disponível em: https://pubmed.ncbi.nlm.nih.gov/18380264/ Acesso em: 20 jul. 2021

COELHO, W. G.; TEDESCO, P. C. A. R. A percepção do outro no ambiente virtual de aprendizagem: presença social e suas implicações para Educação a Distância. Revista Brasileira de Educação, Rio de Janeiro, v. 22, n. 70, p. 609-624, 2017. Disponível

em: 
https://www.scielo.br/j/rbedu/a/RhRHYDDC9ntxQ4mWdPqWcYQ/abstract/?lang=pt Acesso em 20 jul. 2021

COLLINS, A. et al. A longitudinal online interprofessional education experience involving family nurse practitioner students and pharmacy students. Journal Interprofessional Care, London, v. 31, n. 2, p. 218-225, 2017. Disponível em: https://pubmed.ncbi.nlm.nih.gov/28140703/ Acesso em: 20 jul. 2021

CUNHA, M. I. Sala de aula: espaço de inovações e formação docente. In: ENRICONE D, GRILLO M. (Orgs.). Educação superior: vivências e visão de futuro. Porto Alegre: EDIPUCRS 2005, p. 207.

CURREY, J. et al. Developing professional attributes in critical care nurses using Team-Based Learning. Nurse Education in Practice, Edinburg, v. 15, n. 3, p. 232-8, 2015. Disponível em: https://pubmed.ncbi.nlm.nih.gov/25704795/ Acesso em 20 jul. 2021

EL TANTAWI, M. M.: EL KASHLAN, M. K.; SAEED, Y. M. Assessment of the efficacy of second life, a virtual learning environment, in dental education. Journal of Dental Education, Washington, v. 77, n. 12, p. 1639-1652, 2013. Disponível em: https://onlinelibrary. wiley.com/openurl?spage $=1639 \&$ genre $=$ article $\& i s s n=\& d a t e=2013$ \&sid=nlm\%3Apubmed\&issue=12\&volume=77 Acesso em: 20 jul. 2021

FAGEN, A. P.; CROUCH, C. H.; MAZUR, E. Peer Instruction: results from a range of classrooms. The Physics Teacher, Cambridge, v. 40, p. 206-209, 2002. Disponível em: https://aapt.scitation.org/doi/10.1119/1.1474140 Acesso em: 20 jul. 2021

GOUDOURIS, E. S.; GIANNELLA, T. R.; STRUCHINER, M. Tecnologias de informação e comunicação e ensino semipresencial na educação médica. Revista Brasileira de Educação Médica, Brasília, v. 37, n. 3, p. 396-407, 2013. Disponível em: https://www.scielo.br/j/rbem/a/CKJ9hPrqYLR4z5Hj4VKYKcN/abstract/?lang=pt Acesso em: 20 jul. 2021

GUEDES, M. J. P.; ALVES, N. B.; WYSZOMIRSKA, R. M. A. F. Ensino e práticas da fisioterapia aplicada à criança na formação do fisioterapeuta. Fisioterapia em Movimento, Curitiba, v. 26, n. 2, p. 291-305, 2013. Disponível em: https://www.scielo.br/j/fm/a/NwFYgkRn5Wr7Xr8LZtTLzQx/?lang=pt Acesso em: 20 jul. 2021

HILL, J. R.; SONG, L.; WEST, R. E. Social learning theory and web-based learning enviroments: a review of research and discussion of implications. American Journal of Distance Education, v. 23, n. 2, p. 88-103, 2009. Disponível: https://www.tandfonline.com/doi/full/10.1080/08923640902857713. Acesso em: 16 dezembro de 2020.

HRYNCHACK, P.; BATTY, H. The educational theory basis of team-based learning. Medical Teacher, London, v. 34, n. 10, p. 796-801, 2012. Disponível em: https://www.tandfonline.com/doi/abs/10.3109/0142159X.2012.687120?journalCode=i mte20 Acesso em: 20 jul. 2021

HUNT, D. P. et al. The effect of using team learning in an evidence-based medicine course for medical students. Teaching and Learning in Medicine, Hillsdale, v. 15, n. 2, p. 131-139, 2003. Disponível em: https://www.tandfonline.com/doi/abs/10.1207/S15328015TLM1502_11 Acesso em: 20 jul. 2021 
KEAR, K. Social presence in online learning communities Conference or Worshop Item. $2010 . \quad$ Disponível em: https://pdfs.semanticscholar.org/b5b7/6588b5935d2423b14c02cfb116adfa71ca7f.pdf Acesso em: 25 setembro 2020.

KING, A. E.: EGRAS, A. M. A required online course with a public health focus for third professional year pharmacy students. American Journal of Pharmaceutical Education, Alexandria, v. $79, \quad$ n. $5, \quad$ p. 68, 2015. Disponível em: https://www.ncbi.nlm.nih.gov/pmc/articles/PMC4571045/ Acesso em: 20 jul. 2021

KOLES, P. G. et al. The impact of team-based learning on medical students' academic performance. Academic Medicine: journal of the Association American Medical Colleges, Philadelphia, v. 85, n. 11, p. 1739-1745, 2010. Disponível em: https://journals.Iww.com/academicmedicine/Fulltext/2010/11000/The_Impact_of_Tea m_Based_Learning_on_Medical.34.aspx Acesso em: 20 jul. 2021

LARA, F. F.; ROSATTI, L. A.; JOVETTA, R. Análise Swot de duas instituições de ensino superior no estado de São Paulo: potencialidades para a expansão da educação à distância. Revista Brasileira de Administração Científica, Aracajú, v. 7, n. $2, \quad$ p. 127-144, 2016. Disponível em: https://www.sustenere.co/index.php/rbadm/article/view/SPC2179684X.2016.002.0009 Acesso em: 20 jul. 2021

LAU, F. A. et al. Implantação de estratégias de ensino à distância durante o internato: desafios e perspectivas. Revista Brasileira de Educação Médica, Brasília, v. 41, n. 2, p. 269-277, $2017 . \quad$ Disponível em: https://www.scielo.br/j/rbem/a/7WLQyH3tW4xRXgyWj3CQTWd/abstract/?lang=pt Acesso em: 20 jul. 2021

LEIGH, K.; WHITTED, K.; HAMILTON, B. Integration of andragogy into preceptorship. Journal of Adult Education, Helena, v. 44, n. 1, p. 9-17, 2015. Disponível em: https://eric.ed.gov/?id=EJ1072924 Acesso em: 20 jul. 2021

LETASSY, N. A. et al. Using team-based learning in an endocrine module taught across two campuses. American Journal Pharmaceutical Education, Alexandrina, v. $72, \quad$ n.5, p.103, $2008 . \quad$ Disponível em: https://www.ncbi.nlm.nih.gov/pmc/articles/PMC2630128/ Acesso em: 20 jul. 2021

MASTERS, K. Student response to team-based learning and mixed gender teams in an undergraduate medical informatics course. Sultan Qaboos University Medical Journal, Muscat, v. 12, n. 3, 344-351, 2012 . Disponível em: https://www.ncbi.nlm.nih.gov/pmc/articles/PMC3413626/pdf/squmj-12-344.pdf Acesso em: 20 jul. 2021

MAUÉS, O.; SEGENREICH, S.; OTRANTO, C. As políticas de formação de professores: a expansão comprometida. Revista Educação em Questão, Natal, v. 51, n. 37, p.42-72, 2015. Disponível em: https://periodicos.ufrn.br/educacaoemquestao/article/view/7171 Acesso em: 20 jul. 2021

MCINERNEY, M. J.; FINK, L. D. Team-based learning enhances long-term retention and critical thinking in an undergraduate microbial physiology course. Microbiology Educatio, Washington, v. 4, p. 3-12, 2003. Disponível em: https://www.ncbi.nlm.nih.gov/pmc/articles/PMC3633123/ Acesso em: 20 jul. 2021 
MICHAELSEN, L. K.; PARMELEE, D. X.; MCMAHON, K. K. Team based learning for health professions education: a guide to using small groups for improving learning. Sterling: Stylus Publishing, 2008.

MINISTÉRIO DA SAÚDE. Secretaria de Ciência, Tecnologia e Insumos Estratégicos Departamento de Ciência e Tecnologia. Política Nacional de Ciência, Tecnologia e Inovação em Saúde. Série B. Textos Básicos em Saúde. Brasília - DF, 2008.

MITRE, S. M.; SIQUEIRA-BATISTA, R.; GIRARDI-DE-MENDONÇA, J. M.; MORAISPINTO, N. M.; MEIRELLES, C. A. B.; PINTO-PORTO, C.; MOREIRA, T.; HOFFAMANN, L. M. A. Metodologias ativas de ensino-aprendizagem na formação profissional em saúde: debates atuais. Ciência \& Saúde Coletiva, Rio de Janeiro, v. 13 , n. 2, p. 2133-2144, 2008. Acesso em: https://www.scielo.br/j/csc/a/9M86Ktp3vpHgMxWTZXScRKS/?lang=pt Disponível em: 20 jul. 2021

MOORES-DAVIS, T. L. et al. Team-based learning for midwifery education. Journal of Midwifery \& Women`s Health, New York, v. 60, n. 3, p. 291-7, 2015. Disponível em: https://pubmed.ncbi.nlm.nih.gov/25953119/ Acesso em: 20 jul. 2021

MORRISON, F. et al. Developing an online and in-person HIT workforce training program using a team-based learning approach. AMIA Annual Symposium Proceedings, p. 63-71, 2011. Disponível em: https://www.ncbi.nlm.nih.gov/pmc/articles/PMC3243269/ Acesso em: 20 jul. 2021

O'DOHERTY, D. et al. Barriers and solutions to online learning in medical education an integrative review. BMC Medical Education, London, v. 18, n. 1, p. 1-11, 2018.

OLIVEIRA, D. A. A reestruturação do trabalho docente: precarização e flexibilização. Educação \& Sociedade, Campinas, v. 25, n. 89, p. 1127-1144, 2004. Disponível em: https://bmcmededuc.biomedcentral.com/articles/10.1186/s12909-018-1240-0 Acesso em: 20 jul. 2021

OLIVEIRA, E. T.; PICONEZ, S. C. B. Avaliação da educação superior nas modalidades presencial e a distância: análises com base no Conceito Preliminar de Cursos (CPC). Avaliação: Revista da Avaliação da Educação Superior, Campinas, v. 22, n. 30, p. 833-851, 2017. Disponível em: https://www.scielo.br/j/aval/a/dL6pNnxW4bJtjKmLyZqbjgS/abstract/?lang=pt Acesso em: 20 jul. 2021

ONCU, S.; CAKIR, H. Research in online learning environments: priorities and methodologies. Computers \& Education, v. 57, p. 1098-1108, 2011. Disponível em: https://www.sciencedirect.com/science/article/abs/pii/S0360131511000054 Acesso em: 20 jul. 2021

ONRUBIA, J.; COLOMINA, R.; ENGEL, A. As comunidades virtuais de aprendizagem baseadas no trabalho em grupo e na aprendizagem colaborativa. In: COLL, C.; MONEREO, C. (Orgs.). Psicologia da educação virtual: aprender e ensinar com as tecnologias da informação e da comunicação. Porto Alegre: Artmed, 2010.

OUELLETTE, P. S.; BLOUNT, K. Team-based learning in a graduate nurse residency program. J Contin Edu Nurs, New Jersey, v. 46, n. 120, p.572-576, 2015. Disponível em: https://pubmed.ncbi.nlm.nih.gov/26641155/ Acesso em: 20 jul. 2021

PALSOLÉ, S.; AWALK, C. Team-based learning in asynchronous online setting. New Direction Teaching and Learning, v. 116, p.087-95, 2008. Disponível em: 
https://citeseerx.ist.psu.edu/viewdoc/download?doi=10.1.1.616.7488\&rep=rep1\&type $=$ pdf Acesso em: 20 jul. 2021

PARK, H. et al. Effects of team-based learning on perceived teamwork and academic performance in a health assessment subject. Collegian, Subiaco, v. 22, n. 3, p. 299305, 2015. Disponível em: https://pubmed.ncbi.nlm.nih.gov/26552201/ Acesso em: 20 jul. 2021

PEREIRA, V. S.; ANTONIALLI, L. M. Ensino à distância: estratégias de uma universidade federal. Contextus Revista Contemporânea de Economia e Gestão, Fortaleza, v. 9, n. 1, p. 33-48, 2011. Disponível em: http://periodicos.ufc.br/contextus/article/view/32135 Acesso em: 20 jul. 2021

PHILLIPS, J. A.; SCHUMACHER, C.; ARIF, S. Time spent, workload, and student and faculty perceptions in a blended learning environment. American Journal of Pharmaceutical Education, Alexandria, v. 80, n. 6, p. 102, 2016. Disponível em: https://www.ncbi.nlm.nih.gov/pmc/articles/PMC5023973/ Acesso em: 20 jul. 2021

RAYMOND, A. et al. Peer learning a pedagogical approach to enhance online learning: a qualitative exploration. Nurse Educ Today, Edinburgh, n. 44, p. 165-169, 2016. Disponível em: https://pubmed.ncbi.nlm.nih.gov/27429347/ Acesso em: 20 jul. 2021

REIMSCHISEL, T. et al. A systematic review of the published literature on team-based learning in health professions education. Medical Teacher, London, n. 30, p. 1-11, 2017. Disponível em: https://pubmed.ncbi.nlm.nih.gov/28664760/ Acesso em: 20 jul. 2021

SASSO, G. T. M. D.; SOUZA, M. L. Simulação Assistida por Computador: a convergência no processo de educar-cuidar da enfermagem. Texto \& Contexto Enfermagem, Florianópolis, v. 5, n. 2, p. 231-9, 2006. Disponível em: https://www.scielo.br/j/tce/a/NNqKtHYkffspzQfcTz5wqNS/?lang=pt Acesso em: 20 jul. 2021

SCHIBECI, R. et al. Evaluating the use of learning objects in Australian and New Zealand schools. Computers \& Educations, n. 50, p. 271-83, 2008. Disponível em: https://www.sciencedirect.com/science/article/abs/pii/S0360131506001047 Acesso em: 20 jul. 2021

SCHOONHEIM, M.; HEYDEN, R.; WIECHA, J. M. Use of a virtual world computer environment for international distance education: lessons from a pilot project using Second Life. BMC Medical Education, London, v. 14, p. 36, 2014. Disponível em: https://pubmed.ncbi.nlm.nih.gov/24555833/ Acesso em: 20 jul. 2021

SEEFELDT, T. M. et al. A pilot study of interprofessional case discussions for health professions students using the virtual world Second Life. Currents in Pharmacy Teaching and Learning, New York, v. 4, n. 4, p.224-231, 2012. Disponível em: https://www.sciencedirect.com/science/article/abs/pii/S1877129712000470 Acesso em: 20 jul. 2021

SIBLEY, J.; OSTAFICHUK, P. Getting Started with Team-Based Learning. Sterling: Stylus Publishing, LLC; 2014.

TOBASE, L. et al. O design instrucional no desenvolvimento do curso on-line sobre Suporte Básico de Vida. Revista da Escola de Enfermagem da USP, São Paulo, v. $51: e 03288$, 2017. 
https://www.scielo.br/j/reeusp/a/Px7YXPPjgZS5WYzJWKXHB8m/?lang=pt em 20 jul. 2021

VAN DER PUTTEN, M.; VICHIT-VADAKAN, N. A pilot use of team-based learning in graduate public health education. Southeast Asian Journal Tropical Medical Public Health, Bangkok, v. 41, n. 3, p. 743-53, 2010. Disponível em: https://pubmed.ncbi.nlm.nih.gov/20578565/ Acesso em: 20 jul. 2021

WILLETT, L. R.; ROSEVVEAR, G. C.; KIM, S. A trial of team-based versus small-group learning for second-year medical students: does the size of the small group make a difference? Teaching and Learning in Medicine, Hillsdale, v. 23, n. 1, p. 28-30, 2011. Disponível em: https://pubmed.ncbi.nlm.nih.gov/21240779/ Acesso em: 20 jul. 2021

WOO, Y.; REEVES, T. C. Meaningful interaction in web-based learning: a social constructivist interpretation. Internet and Higher Education, v. 10, p. 15-25, 2007. Disponível

em:

https://www.sciencedirect.com/science/article/abs/pii/S1096751606000753 Acesso em: 20 jul. 2021

Recebido: $17 / 12 / 2020$

Aprovado: 29/07/2021

Como citar: AMORIM, J. S. C.; KNEIPP, R. E. Adaptação do método team-based learning para o modelo mediado por tecnologias virtuais de aprendizagem. Educitec - Revista de Estudos e

Pesquisas sobre Ensino Tecnológico, v. 7, e161721, 2021.

Contribuição de autoria:

Juleimar Soares Celho de Amorim: Conceituação, investigação, escrita (rascunho original), escrita (revisão e edição).

Ricardo Esteves Kneipp: Administração do projeto, escrita (rascunho original), escrita (revisão e edição).

Direito autoral: Este artigo está licenciado sob os termos da Licença Creative CommonsAtribuição 4.0 Internacional

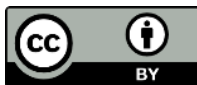

